\title{
GSTP1 Ile105 Val and XRCC1 Arg399Gln gene polymorphisms contribute to the clinical outcome of patients with advanced non-small cell lung cancer
}

\author{
L. Bu ${ }^{1,2}$, L.B. Zhang ${ }^{1}$, X. Mao ${ }^{1}$ and P. Wang ${ }^{1}$ \\ ${ }^{1}$ Department of Thoracic Surgery, First People's Hospital of Yunnan Province, \\ Kunhua Hospital Affiliated to Kunming University of Science and Technology, \\ Kunming, China \\ ${ }^{2}$ Medical Faculty of Science, Kunming University of Science and Technology, \\ Kunming, China
}

Corresponding author: P. Wang

E-mail: wangping_kmu@163.com

Genet. Mol. Res. 15 (2): gmr.15027611

Received September 10, 2015

Accepted December 22, 2015

Published June 3, 2016

DOI http://dx.doi.org/10.4238/gmr.15027611

\begin{abstract}
Glutathione S-transferase P1 (GSTP1) and X-ray repair cross-complementing group 1 (XRCC1) genetic variations may influence the efficacy of chemotherapy in various cancers. We investigated the possible roles of GSTP1 Ile105Val and XRCC1 Arg194Trp, and $\mathrm{Arg} 399 \mathrm{Gln}$ gene polymorphisms in the prognosis of advanced non-small cell lung carcinoma (NSCLC) patients with cisplatin-based chemotherapy. Between January 2010 and December 2012, this study consecutively recruited 141 patients with advanced NSCLC from the First People's Hospital of Yunnan Province. Logistic regression analysis showed that individuals carrying the GG genotype were associated with a better response to chemotherapy than those with the wide-type genotype, with an adjusted odds ratio (95\% confidence interval, CI) of 4.07 (1.06-25.06). Moreover, we observed that the AA genotype of
\end{abstract}


XRCC1 Arg399Gln was correlated with a greater complete response + partial response to chemotherapy than that with the GG genotype (odds ratio $=2.71,95 \% \mathrm{CI}=1.13-10.08$ ). . Based on the Cox hazard proportional model, the GG genotype of GSTP1 Ile105Val was found to be associated with a lower risk of death from all causes as compared to that with the AA genotype (hazard ratio $=0.07,95 \% \mathrm{CI}=0.01-0.34$ ). In summary, we suggest that GSTP1 Ile105Val and XRCC1 Arg399Gln polymorphisms could influence the response to chemotherapy and survival of advanced NSCLC.

Key words: Clinical outcome; GSTP1 Ile105Val; XRCC1 Arg194Trp; Non-small cell lung carcinoma; XRCC1 Arg399Gln

\section{INTRODUCTION}

Lung cancer is a worldwide common malignancy that arises from lung tissue and has become one of the most common factor leading to human deaths (IARC, 2012). Non-small cell lung cancer (NSCLC) and small cell lung cancer are the two types for lung cancer based on the histological characteristics, in which NSCLC accounts for about $80 \%$ of lung cancer (Beadle et al., 2014). NSCLC is typically diagnosed at an advanced stage, and the 5-year survival rate for lung cancer is approximately $15 \%$ (Henley et al., 2014). Current studies have shown that the prognosis of NSCLC can be attributed to a variety of environmental and genetic factors, such as TNM stage, therapy method, and genetic factors (Gou et al., 2015; Ramalingam and Khuri, 2015; Wei et al., 2015). Therefore, it is important to understand the role of various cancer-related genes in the development and prognosis of NSCLC, so as to identify the prognosis markers for NSCLC.

Previous studies have reported that glutathione S-transferase P1 (GSTP1) and X-ray repair cross-complementing group $1(X R C C 1)$ genetic variations may influence the efficacy of chemotherapy in various cancers, such as colon cancer, gastric cancer, and ovarian cancer (Khrunin et al., 2010; Ji et al., 2013; Lai et al., 2013; Zaanan et al., 2014). Currently, many studies reported the role of GSTP1 and XRCCl genetic polymorphisms in the clinical outcome of NSCLC, but the results are inconclusive. In this study, we investigated the possible roles of GSTP1 Ile105 Val and XRCC1 Arg194Trp, and Arg399Gln gene polymorphisms in the prognosis of advanced NSCLC patients with cisplatin-based chemotherapy.

\section{MATERIAL AND METHODS}

Between January 2010 and December 2012, this study consecutively recruited 141 patients with advanced NSCLC from the First People's Hospital of Yunnan Province. All the NSCLC patients were histopathologically confirmed and newly diagnosed. All patients with advanced NSCLC did not receive anticancer therapies before surgery and had adequate hematology, renal, and liver function with an Eastern Cooperative Oncology Group performance status 0 or 1 . Patients that receive anticancer therapies prior to enrollment and have acute and chronic infection disease were excluded from this study. The patients were routinely followed up every 4 weeks during disease progression.

All the advanced NSCLC patients underwent the cisplatin-based chemotherapy after 
enrollment. Response to chemotherapy of the NSCLC patients was assessed according to RECIST criteria in 2000 (Duffaud and Therasse, 2000). A good response to chemotherapy was defined as a complete response (CR) and partial response (PR) to chemotherapy, and a poor response to chemotherapy was defined as a stable disease (SD) and progressive disease (PD). The overall survival was used as the endpoint index, and it was calculated by the time between the date of undergoing chemotherapy and the date of death from any cause or the end of the study. Routine follow-ups of all patients were ended in December 2014, and patients were followed up every 4 weeks through telephone interview.

\section{DNA extraction and genotyping}

DNA was extracted from peripheral blood lymphocytes using the Qiagen blood mini-kit (Qiagen, Hilden, Germany) following the manufacturer instructions. The GSTP1 Ile105 Val and XRCC1 Arg194Trp and Arg399Gln genotypes were determined by polymerase chain reaction-restriction fragment length polymorphism (PCR-RFLP) assay. DNA samples were amplified using two different primer pairs specific for GSTP1 Ile105Val and XRCC1 Arg194Trp and Arg399Gln genes. The forward and reverse primers were designed using the Sequenom Assay Design 3.1 software (San Diego, CA, USA). PCR was conducted with an initial melting step of $94^{\circ} \mathrm{C}$ for $5 \mathrm{~min}$, followed by 30 cycles of denaturation at $94^{\circ} \mathrm{C}$ for 45 $\mathrm{s}$, annealing at $62^{\circ} \mathrm{C}$ for $45 \mathrm{~s}$, extension at $72^{\circ} \mathrm{C}$ for $45 \mathrm{~s}$, and a final extension at $72^{\circ} \mathrm{C}$ for 5 min. PCR amplification was checked by using $1.5 \%$ agarose gel electrophoresis. Further, the amplified products were digested with $5 \mathrm{U}$ of each restriction enzyme. Around $10 \%$ of the samples containing all genotypes for GSTP1 Ile105 Val and XRCC1 Arg194Trp and Arg399Gln genes were repeated for PCR-RFLP polymorphism and all were matched to their genotype.

\section{Statistical analysis}

The association between the response to chemotherapy and GSTP1 Ile105Val, XRCC1 Arg 194Trp, and Arg399Gln polymorphisms was tested using odds ratio (OR) and 95\% confidence interval $(95 \% \mathrm{CI})$ in logistic regression analysis, and the wide-type genotype was taken as a reference group. The overall survival time of advanced NSCLC patients carrying GSTP1 Ile105Val, XRCC1 Arg194Trp, and Arg399Gln polymorphisms were tested using the Kaplan-Meier method. Cox hazard proportional model was used to calculate the overall survival of advanced NSCLC. The results are reported using hazard ratio and 95\%CI with adjustments for potential confounding factors. All P values were based on a 2-tailed test and $\mathrm{P}$ $<0.05$ was considered significant. The SPSS software version 20.0 (SPSS Inc., Chicago, IL, USA) was carried out for statistical analysis.

\section{RESULTS}

The mean age of patients with advanced NSCLC was $55.95 \pm 7.83$ years (Table 1). This study was comprised of $41(29.08 \%)$ females and 100 (70.92) males in the present study. In advanced NSCLC patients, $93(65.96 \%)$ were smokers, 51 (36.17\%) were drinkers, 62 $(43.97 \%)$ were at III TNM stage, $79(56.03 \%)$ were at IV TNM stage, $62(43.97 \%)$ were adenocarcinoma type, $79(56.03 \%)$ were squamous carcinoma type, $71(50.35 \%)$ showed $\mathrm{CR}+\mathrm{PR}$ to chemotherapy, and $70(49.65 \%)$ showed SD+PD to chemotherapy. 
Table 1. Demographic and clinical characteristics of the recruited patients and control subjects.

\begin{tabular}{l|c|c|c}
\hline Variables & Patients & $\%$ & Survival time \\
\hline Age (years) & $55.95 \pm 7.83$ & 65.96 & $21.10 \pm 1.47$ \\
\hline$<60$ & 93 & 34.04 & $21.94 \pm 1.52$ \\
\hline$\geq 60$ & 48 & & $20.52 \pm 2.05$ \\
\hline Gender & 41 & 70.92 & $21.38 \pm 1.40$ \\
\hline Female & 100 & & \\
\hline Male & & 34.04 & $21.48 \pm 1.72$ \\
\hline Smoking status & 48 & 65.96 & $21.60 \pm 1.53$ \\
\hline Never & 93 & & $21.51 \pm 1.41$ \\
\hline Ever & 90 & 63.83 & $21.82 \pm 2.04$ \\
\hline Drinking status & 51 & 36.17 & \\
\hline Never & & & $22.53 \pm 1.68$ \\
\hline Ever & 62 & 43.97 & $20.24 \pm 1.58$ \\
\hline TNM stage & 79 & 56.03 & \\
\hline III & & & \\
\hline IV & 62 & 43.97 & $20.83 \pm 1.71$ \\
\hline Histology & 79 & 56.03 & $22.14 \pm 1.59$ \\
\hline Sdenocarcinoma & & & \\
\hline Response to chemotherapy & 71 & 50.35 & $25.52 \pm 1.67$ \\
\hline CR+PR & 70 & 49.65 & $17.55 \pm 1.48$ \\
\hline SD+PD & & & \\
\hline
\end{tabular}

The association between GSTP1 Ile105Val, XRCC1 Arg194Trp, and Arg399Gln and the response to chemotherapy in advanced NSCLC is shown in Table 2. Based on logistic regression analysis, we observed that the GG genotype of GSTP1 Ile105Val were associated with a greater $\mathrm{CR}+\mathrm{PR}$ response to chemotherapy compared to the common genotype, and the adjusted OR $(95 \% \mathrm{CI})$ was 4.07 (1.06-25.06). Moreover, the AA genotype of XRCC1 Arg399Gln was associated with a greater $\mathrm{CR}+\mathrm{PR}$ response to chemotherapy compared to the wide-type genotype $(\mathrm{OR}=2.71,95 \% \mathrm{CI}=1.13-10.08)$. However, we did not observe a significant correlation between the XRCC1 Arg194Trp polymorphism and the response to chemotherapy in advanced NSCLC patients.

Table 2. Association between GSTP1 Ile105Val, XRCC1 Arg194Trp, and Arg399Gln and response to chemotherapy in advanced NSCLC.

\begin{tabular}{l|c|c|c|c|c|c|c|c}
\hline Genes & $\mathrm{N}$ & $\%$ & Good response & $\%$ & Poor response & $\%$ & OR (95\%CI) $^{1}$ & P value \\
\hline GSTP1 Ile105Val & & & & & & & & \\
\hline AA & 66 & 46.81 & 28 & 39.44 & 38 & 54.29 & 1.0 (Ref.) & - \\
\hline AG & 63 & 44.68 & 34 & 47.89 & 29 & 41.43 & $1.59(0.75-3.38)$ & 0.19 \\
\hline GG & 12 & 8.51 & 9 & 12.68 & 3 & 4.29 & $4.07(1.06-25.06)$ & 0.03 \\
\hline XRCC1 Arg194Trp & & & & & & & & \\
\hline CC & 64 & 45.39 & 29 & 40.85 & 35 & 50.00 & $1.0($ Ref.) & - \\
\hline CT & 58 & 41.13 & 31 & 43.66 & 27 & 38.57 & $1.39(0.64-3.01)$ & 0.37 \\
\hline TT & 19 & 13.48 & 11 & 15.49 & 8 & 11.43 & $1.66(0.52-5.42)$ & 0.34 \\
\hline XRCC1 Arg399Gln & & & & & & & & \\
\hline GG & 61 & 43.26 & 26 & 36.62 & 35 & 50.00 & $1.0($ Ref.) & - \\
\hline GA & 53 & 37.59 & 24 & 33.80 & 29 & 41.43 & $1.11(0.50-2.50)$ & 0.78 \\
\hline AA & 27 & 19.15 & 21 & 29.58 & 6 & 8.57 & $2.71(1.13-10.08)$ & 0.01 \\
\hline
\end{tabular}

${ }^{1}$ Ajusted for age, gender, smoking status, drinking status, TNM stage, and histology.

The median overall survival time was 23.55 months. At the end of the study, 112 advanced NSCLC patients died from all causes. The GG genotype of GSTP1 Ile105Val was associated with a longer overall survival time compared to the wide-type genotype ( $\mathrm{P}$ value for log-rank test $=0.001$; Figure 1$)$. After adjusting for age, gender, smoking status, drinking 
status, TNM stage, and histology, we observed that the GG genotype of GSTP1 Ile105Val was correlated with a lower risk of death compared to the wide type genotype (hazard ratio $=0.07$, 95\%CI = 0.01-0.34) (Table 3). The XRCC1 Arg194Trp, and Arg399Gln polymorphisms did not exhibit a significant association with the survival of advanced NSCLC patients.

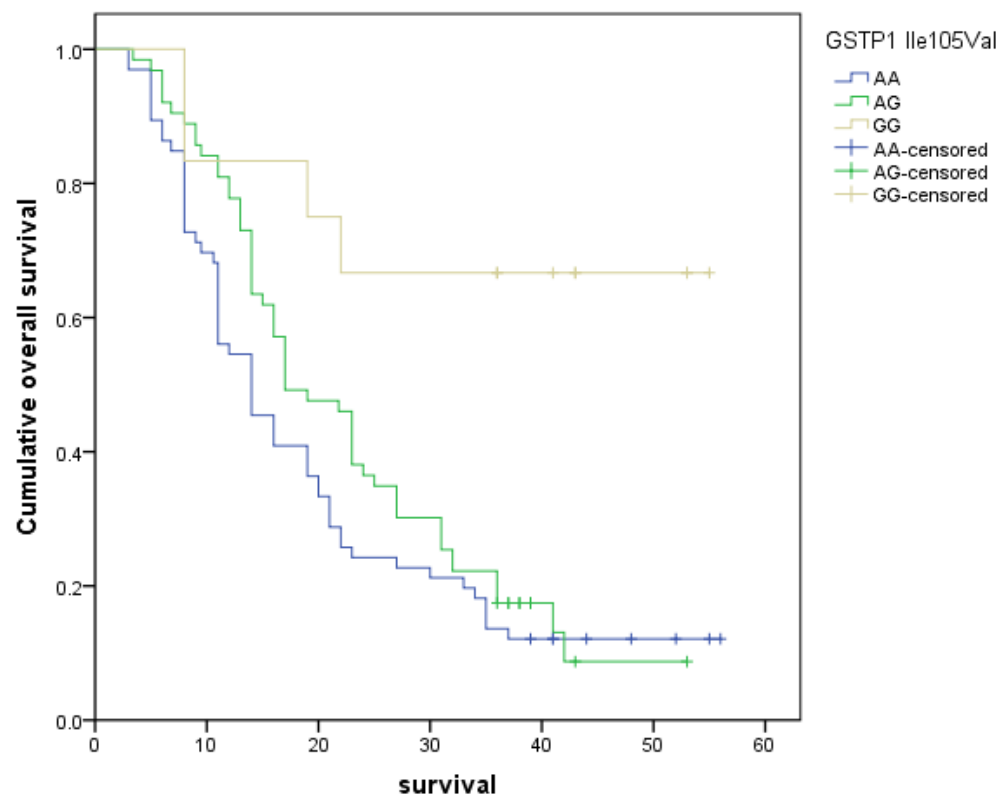

Figure 1. Cumulative overall survival of advanced NSCLC by the GSTP1 Ile105Val polymorphism.

Table 3. Association between GSTP1 Ile105Val, XRCC1 Arg194Trp, and Arg399Gln and overall survival of patients with advanced NSCLC.

\begin{tabular}{|c|c|c|c|c|c|c|c|c|}
\hline Genes & $\mathrm{N}$ & $\%$ & Death & $\%$ & Alive & $\%$ & Adjusted HR $(95 \% \mathrm{CI})^{1}$ & $\mathrm{P}$ value \\
\hline \multicolumn{9}{|c|}{ GSTP1 Ile105 Val } \\
\hline AA & 66 & 46.81 & 58 & 50.00 & 8 & 30.77 & 1.0 (Ref.) & - \\
\hline $\mathrm{AG}$ & 63 & 44.68 & 54 & 46.55 & 9 & 34.62 & $0.83(0.26-2.62)$ & 0.72 \\
\hline GG & 12 & 8.51 & 4 & 3.45 & 8 & 30.77 & $0.07(0.01-0.34)$ & $<0.001$ \\
\hline \multicolumn{9}{|c|}{ XRCC1 Arg194Trp } \\
\hline $\mathrm{CC}$ & 64 & 45.39 & 56 & 48.28 & 8 & 30.77 & 1.0 (Ref.) & - \\
\hline CT & 58 & 41.13 & 45 & 38.79 & 13 & 50.00 & $0.49(0.16-1.43)$ & 0.15 \\
\hline TT & 19 & 13.48 & 15 & 12.93 & 4 & 15.38 & $0.54(0.12-2.79)$ & 0.35 \\
\hline \multicolumn{9}{|c|}{ XRCC1 Arg399Gln } \\
\hline GG & 61 & 43.26 & 51 & 43.97 & 10 & 38.46 & 1.0 (Ref.) & - \\
\hline GA & 53 & 37.59 & 37 & 31.90 & 16 & 61.54 & $0.45(0.16-1.21)$ & 0.08 \\
\hline AA & 27 & 19.15 & 19 & 16.38 & 8 & 30.77 & $0.47(0.14-1.59)$ & 0.16 \\
\hline
\end{tabular}

${ }^{1}$ Ajusted for age, gender, smoking status, drinking status, TNM stage, and histology.

\section{DISCUSSION}

The present case-control study investigated the role of the GSTP1 Ile105 Val, XRCC1 Arg 194Trp, and Arg399Gln polymorphisms in the response to cisplatin-based chemotherapy and the survival of advanced NSCLC patients. We found that the GSTP1 IIe105Val and XRCC1 
Arg399Gln genetic polymorphisms promoted the response to chemotherapy and the survival time in the advanced NSCLC patients.

The amino acid substitution changes of GSTP1 Ile105Val gene could cause hydroliphobicity of amino acids, which alters the enzymatic stability and catalytic function. The GSTP1 Ile105 Val polymorphism is reported to be associated with the efficacy of chemotherapy in various cancers, such as breast cancer, bladder cancer, osteosarcoma, non-small cell lung cancer, and gastric cancer (Deng et al., 2015a,b; Liu et al., 2013, 2015; Pu et al., 2015; Yuan et al., 2015). Liu et al. (2013) revealed that GSTP1 IIe105Val and XRCC1 Arg399Gln polymorphisms may contribute to the clinical outcome of gastric cancer patients receiving oxaliplatin-based adjuvant chemotherapy. Liu et al. (2015) carried out a study in a Chinese advanced NSCLC and reported that GSTP1 IIe105Val and XRCC1 Arg399Gln polymorphisms might influence the clinical outcome of patients with advanced NSCLC receiving cisplatinbased chemotherapy. Deng et al. (2015b) suggested that patients with the GSTP1 IIe105Val Val allele were associated with a favorable recurrence-free survival of advanced NSCLC. $\mathrm{Pu}$ et al. (2015) conducted a meta-analysis with six studies involving 898 participants, and indicated that GSTP1 polymorphisms may influence the prognosis of osteosarcoma patients treated with chemotherapy. Yuan et al. (2015) carried out a study in a Chinese population and reported that the GG genotype of GSTP1 Ile105Val was significantly correlated with better response to chemotherapy and longer survival time in breast cancer.

Currently, some studies have investigated the association between genetic polymorphisms in the GSTP1 IIe105Val region and the prognosis of advanced NSCLC, but the results are inconclusive (Ada et al., 2010; Zhou et al., 2011; Ke et al., 2012; Lv et al., 2014; Yang and Zhao, 2014; Deng et al., 2015; Han et al., 2015; Liu et al., 2015). Individuals with GSTP1 gene polymorphisms display a reduced ability to detoxify drug metabolites, thus promoting the overall survival of NSCLC (Lu et al., 2006; Ke et al., 2012; Lv et al., 2014; Deng et al., 2015; Han et al., 2015). Four studies reported that the Val allele of GSTP1 IIe105Val obtained a better response to chemotherapy and a better survival time when compared to IIe allele in Asian population (Ada et al., 2010; Zhou et al., 2011; Ke et al., 2012; Lv et al., 2014; Han et al., 2015). Two studies suggested that the Val allele of GSTP1 IIe105Val was more highly correlated with a poor response to chemotherapy than those carrying the IIe genotype (Deng et al., 2015; Liu et al., 2015). However, one study did not find a significant association between GSTP1 IIe105 Val polymorphism and clinical outcome of advanced NSCLC (Ada et al., 2010). A recent meta-analysis involving nine studies suggested that GSTP1 IIe105Val polymorphisms could predict the treatment response of the platinum-based chemotherapy in NSCLC patients. Our study reported the similar results with previous studies.

The function of DNA damage may be affected by cisplatin, and the DNA repair capacity may be correlated with the efficiency and toxicity of cisplatin-based chemotherapy. $X R C C 1$ gene polymorphisms are reported to be correlated with the treatment outcome of cisplatin-based chemotherapy in several kinds of cancers, such as gastric cancer, colorectal cancer, nasopharyngeal cancer, ovarian cancer, pancreatic cancer, and esophageal cancer (Warnecke-Eberz et al., 2009; Giovannetti et al., 2011; Miao et al., 2012; Chen et al., 2013; Cao et al., 2014; Wu et al., 2014). Currently, several studies have reported the association between XRCC1 genetic polymorphisms and treatment outcome of advanced NSCLC (Yuan et al., 2006; Wang et al., 2004, 2008; Sun et al., 2009; Li et al., 2011; Ke et al., 2012; Han et al., 2015). Three studies were carried out and reported a significant association between the XRCC1 Arg194Trp polymorphism and clinical response to platinum-based chemotherapy in 
advanced NSCLC (Wang et al., 2004; Yuan et al., 2006; Ke et al., 2012). Five studies have suggested an significant association of the XRCC1 Arg399Gln polymorphism and clinical outcome of advanced NSCLC patients in Asian populations (Wang et al., 2008; Sun et al., 2009; Li et al., 2011; Ke et al., 2012; Han et al., 2015). Our study only reported that the XRCC1 Arg399Gln polymorphism could influence the clinical response to platinum-based chemotherapy. Further large scale studies are needed to confirm our results.

In summary, we suggest that GSTP1 Ile 105Val and XRCC1 Arg399Gln were associated with a better response to chemotherapy and longer survival of advanced NSCLC compared to the wild-type genotype. Therefore, studies including larger sample sizes must be performed to confirm our findings.

\section{Conflicts of interest}

The authors declare no conflict of interest.

\section{ACKNOWLEDGMENTS}

We thank the patients who agreed to provide their blood for study analysis.

\section{REFERENCES}

Ada AOC, C Kunak S, Hancer F, Bilgen S, et al. (2010). CYP and GST polymorphisms and survival in advanced nonsmall cell lung cancer patients. Neoplasma 57: 512-521. http://dx.doi.org/10.4149/neo $2010 \quad 06 \quad 512$

Beadle J, Coats A, Ho G, Prabhash K, et al. (2014). 487 psurvival and quality of life data from the act one randomised, double-blind, placebo-controlled, phase ii study of espindolol for the treatment and prevention of cachexia in patients with stage iii/iv non-small cell lung cancer or colorectal cancer. Ann. Oncol. 25: iv162-iv163.

Cao Z, Song J, Wang J, Guo X, et al. (2014). Association between polymorphisms in XRCC1 gene and treatment outcomes of patients with advanced gastric cancer: a systematic review and meta-analysis. PLoS One 9: e85357. http://dx.doi. org/10.1371/journal.pone.0085357

Chen C, Wang F, Wang Z, Li C, et al. (2013). Polymorphisms in ERCC1 C8092A predict progression-free survival in metastatic/recurrent nasopharyngeal carcinoma treated with cisplatin-based chemotherapy. Cancer Chemother. Pharmacol. 72: 315-322. http://dx.doi.org/10.1007/s00280-013-2196-8

Deng JH, Deng J, Shi DH, Ouyang XN, et al. (2015). Clinical outcome of cisplatin-based chemotherapy is associated with the polymorphisms of GSTP1 and XRCC1 in advanced non-small cell lung cancer patients. Clin. Transl. Oncol. 17: 720-726. http://dx.doi.org/10.1007/s12094-015-1299-6

Duffaud F and Therasse P (2000). New guidelines to evaluate the response to treatment in solid tumors. Bull. Cancer 87: 881-886.

Giovannetti E, Pacetti P, Reni M, Leon LG, et al. (2011). Association between DNA-repair polymorphisms and survival in pancreatic cancer patients treated with combination chemotherapy. Pharmacogenomics 12: 1641-1652. http://dx.doi. org/10.2217/pgs.11.109

Gou LY, Niu FY, Wu YL and Zhong WZ (2015). Differences in driver genes between smoking-related and non-smokingrelated lung cancer in the Chinese population. Cancer 121 (Suppl 17): 3069-3079. http://dx.doi.org/10.1002/ $\underline{\text { cncr.29531 }}$

Han B, Guo Z, Ma Y, Kang S, et al. (2015). Association of GSTP1 and XRCC1 gene polymorphisms with clinical outcome of advanced non-small cell lung cancer patients with cisplatin-based chemotherapy. Int. J. Clin. Exp. Pathol. 8: 4113-4119.

Henley SJ, Richards TB, Underwood JM, Eheman CR, et al.; Centers for Disease Control and Prevention (CDC) (2014). Lung cancer incidence trends among men and women - United States, 2005-2009. MMWR Morb. Mortal. Wkly. Rep. 63: $1-5$.

International Agency for Research on Cancer (2012). Lung Cancer. Estimated Incidence, Mortality and Prevalence Worldwide in 2012. http://globocan.iarc.fr/Pages/fact_sheets_cancer.aspx. AccessedAugust 1, 2015. 
Ji M, Xu B, Jiang JT, Wu J, et al. (2013). Relationship between glutathione S-transferase P1 (GSTP1), X-ray repair cross complementing group 1 (XRCC1) and 5,10-methylenetetrahydrofolate reductase (5,10-MTHFR) gene polymorphisms and response to chemotherapy in advanced gastric cancer. Onkologie 36: 335-340. http://dx.doi. org $/ 10.1159 / 000351260$

Ke HG, Li J, Shen Y, You QS, et al. (2012). Prognostic significance of GSTP1, XRCC1 and XRCC3 polymorphisms in non-small cell lung cancer patients. Asian Pac. J. Cancer Prev. 13: 4413-4416. http://dx.doi.org/10.7314/ $\underline{\text { APJCP.2012.13.9.4413 }}$

Khrunin AV, Moisseev A, Gorbunova V and Limborska S (2010). Genetic polymorphisms and the efficacy and toxicity of cisplatin-based chemotherapy in ovarian cancer patients. Pharmacogenomics J. 10: 54-61. http://dx.doi.org/10.1038/ tpj.2009.45

Lai CY, Hsieh LL, Sung FC, Tang R, et al. (2013). Tumor site- and stage-specific associations between allelic variants of glutathione S-transferase and DNA-repair genes and overall survival in colorectal cancer patients receiving 5-fluorouracil-based chemotherapy. PLoS One 8: e69039. http://dx.doi.org/10.1371/journal.pone.0069039

Li Y, Huang XE, Jin GF, Shen HB, et al. (2011). Lack of any relationship between chemotherapy toxicity in non-small cell lung cancer cases and polymorphisms in XRCC1 codon 399 or XPD codon 751. Asian Pac. J. Cancer Prev. 12: 739-742.

Liu JY, Liu QM, Li LR and Kang S (2015). Association of GSTP1 and XRCC1 gene polymorphisms with clinical outcomes of patients with advanced non-small cell lung cancer. Genet. Mol. Res. 14: 10331-10337. http://dx.doi. org/10.4238/2015.August.28.19

Liu YP, Ling Y, Qi QF, Zhang YP, et al. (2013). Genetic polymorphisms of ERCC1118, XRCC1399 and GSTP1105 are associated with the clinical outcome of gastric cancer patients receiving oxaliplatinbased adjuvant chemotherapy. Mol. Med. Rep. 7: 1904-1911.

Lu C, Spitz MR, Zhao H, Dong Q, et al. (2006). Association between glutathione S-transferase pi polymorphisms and survival in patients with advanced nonsmall cell lung carcinoma. Cancer 106: 441-447. http://dx.doi.org/10.1002/ cncr.21619

Lv H, Han T, Shi X, Yao Y, et al. (2014). Genetic polymorphism of GSTP1 and ERCC1 correlated with response to platinum-based chemotherapy in non-small cell lung cancer. Med. Oncol. 31: 86. http://dx.doi.org/10.1007/s12032$\underline{014-0086-5}$

Miao J, Zhang X, Tang QL, Wang XY, et al. (2012). Prediction value of XRCC 1 gene polymorphism on the survival of ovarian cancer treated by adjuvant chemotherapy. Asian Pac. J. Cancer Prev. 13: 5007-5010. http://dx.doi. org/10.7314/APJCP.2012.13.10.5007

Pu F, Chen F, Chen S, Wang B, et al. (2015). Association between GSTP1 polymorphisms and prognosis of osteosarcoma in patients treated with chemotherapy: a meta-analysis. Onco Targets Ther. 8: 1835-1842.

Ramalingam SS and Khuri FR (2015). Lung cancer in China: The new frontier? Cancer 121 (Suppl 17): 3058-3060. http:// dx.doi.org/10.1002/cncr.29569

Sun X, Li F, Sun N, Shukui Q, et al. (2009). Polymorphisms in XRCC1 and XPG and response to platinum-based chemotherapy in advanced non-small cell lung cancer patients. Lung Cancer 65: 230-236. http://dx.doi.org/10.1016/j. lungcan.2008.11.014

Wang ZH, Miao XP, Tan W, Zhang XR, et al. (2004). [Single nucleotide polymorphisms in XRCC1 and clinical response to platin-based chemotherapy in advanced non-small cell lung cancer]. Ai Zheng 23: 865-868.

Wang Z, Xu B, Lin D, Tan W, et al. (2008). XRCC1 polymorphisms and severe toxicity in lung cancer patients treated with cisplatin-based chemotherapy in Chinese population. Lung Cancer 62: 99-104. http://dx.doi.org/10.1016/j. lungcan.2008.02.019

Warnecke-Eberz U, Vallböhmer D, Alakus H, Kütting F, et al. (2009). ERCC1 and XRCC1 gene polymorphisms predict response to neoadjuvant radiochemotherapy in esophageal cancer. J. Gastrointest. Surg. 13: 1411-1421. http:// dx.doi.org/10.1007/s11605-009-0881-z

Wei R, DeVilbiss FT and Liu W (2015). Genetic Polymorphism, Telomere Biology and Non-Small Lung Cancer Risk. $J$. Genet. Genomics 42: 549-561. http://dx.doi.org/10.1016/j.jgg.2015.08.005

$\mathrm{Wu} \mathrm{H}, \mathrm{Xu} \mathrm{C}$, Chen G and Wang J (2014). X-ray repair cross-complementing 1 polymorphism and prognosis of platinumbased chemotherapy in gastric and colorectal cancer: a meta-analysis. J. Gastroenterol. Hepatol. 29: 926-933. http:// dx.doi.org/10.1111/jgh.12444

Yang Z and Zhao J (2015). Effect of APE1 and XRCC1 gene polymorphism on susceptibility to hepatocellular carcinoma and sensitivity to cisplatin. Int. J. Clin. Exp. Med. 8: 9931-9936.

Yuan P, Miao XP, Zhang XM, Wang ZH, et al. (2006). [XRCC1 and XPD genetic polymorphisms predict clinical responses to platinum-based chemotherapy in advanced non-small cell lung cancer]. Zhonghua Zhong Liu Za Zhi 28: 196-199.

Genetics and Molecular Research 15 (2): gmr.15027611

CFUNPEC-RP www.funpecrp.com.br 
Yuan P, Yuan L, Xu BL, Wang CZ, et al. (2015). Predictive potential role of glutathione S-transferases polymorphisms in response to chemotherapy and breast cancer prognosis. Genet. Mol. Res. 14: 16675-16681. http://dx.doi. org/10.4238/2015.December.11.15

Zaanan A, Dalban C, Emile JF, Blons H, et al. (2014). ERCC1, XRCC1 and GSTP1 Single Nucleotide Polymorphisms and Survival of Patients with Colon Cancer Receiving Oxaliplatin-Based Adjuvant Chemotherapy. J. Cancer 5: 425-432. http://dx.doi.org/10.7150/jca.8594

Zhou F, Yu Z, Jiang T, Lv H, et al. (2011). Genetic polymorphisms of GSTP1 and XRCC1: prediction of clinical outcome of platinum-based chemotherapy in advanced non-small cell lung cancer (NSCLC) patients. Swiss Med. Wkly. 141: w13275. 